\title{
MIMO Nullforming with RVQ Limited Feedback and Channel Estimation Errors
}

\author{
D. Richard Brown III \\ Dept. of Electrical and Computer Eng. \\ Worcester Polytechnic Institute \\ 100 Institute Rd, Worcester, MA 01609 \\ drb@wpi.edu
}

\author{
David J. Love \\ School of Electrical and Computer Eng. \\ Purdue University \\ West Lafayette, IN 47907 \\ djlove@ecn.purdue.edu
}

\begin{abstract}
This paper explores limited feedback nullforming techniques based on random vector quantization (RVQ) with and without receiver coordination. The availability of receiver coordination affects the type and amount of feedback required to select an appropriate precoding vector. Approximate upper and lower bounds are developed for the mean received power at primary receivers with and without receiver coordination. Numerical results confirm the analysis and show that the channel estimation errors effectively establish a floor on the achievable performance of RVQ nullforming. The size of the RVQ codebook can be selected to approach this floor without excessive overhead.
\end{abstract}

Index Terms - antenna arrays, nullforming, zero-forcing, limited feedback, random vector quantization, MIMO communication, interference mitigation, channel estimation error

\section{INTRODUCTION}

Beamforming and nullforming require channel state knowledge at the transmitter to achieve a desired directivity pattern. It is often necessary to estimate the channels at the receiver and provide feedback to the transmitter to facilitate multiantenna transmission with a desired directivity pattern [1]. Since feedback creates overhead, several limited-rate feedback techniques have been developed recently for multi-input multi-output (MIMO) and multi-input single-output (MISO) systems, for example see [2]-[10] and the references therein.

Among the various limited-rate feedback techniques, random vector quantization (RVQ) is appealing since the codebook, known to both the transmitter and the receiver, is randomly generated independently of the channel realization. RVQ was first proposed in [11], [12] and was shown to be asymptotically optimal for beamforming as $M, N \rightarrow \infty$ in [13], [14] where $M$ is the number of transmit antennas and $N$ is the number of precoding vectors in the RVQ codebook. RVQ has received further analysis in [15]-[17]. The performance of RVQ for a MISO beamforming system was studied in [18] where it was shown that the beamforming loss due to RVQ was on the order of $N^{-\frac{1}{M-1}}$.

In this paper, we consider MIMO nullforming with RVQ limited feedback in systems with and without receiver coordination. Our focus is on a single-stream scenario where one or more "primary" receivers provide feedback to the transmitter

This work was supported by the National Science Foundation awards CCF1302104 and CCF-1319458. to facilitate nullforming toward these receivers. "Secondary" receivers in the network do not provide feedback to the transmitter and experience channels statistically equivalent to a single-antenna fading channel. Examples of applications under this model include (i) jamming, (ii) cognitive radio, and (iii) overlay networks. Since the statistics of the received power at the secondary users is independent of the transmitter's normalized precoding vector, our analysis focuses on quantifying the mean received null power at the primary receivers as a function of the number of transmit antennas $M$, number of primary receivers $K<M$, the RVQ codebook size $N$, and the variance of the channel estimation errors $\sigma^{2}$.

Initial results on this problem were presented in [19], which developed upper bounds on the mean received power when each receiver has a perfect estimate of its channel. In the absence of channel estimation error, a zero-forcing precoder with unquantized feedback can achieve perfect nulls such that the power at the primary receivers is exactly zero. A system using RVQ limited feedback nullforming, however, can only achieve nulls of limited depth due to the inherent quantization of the precoding vectors. We present analytical results showing that, for a system with an $M$-antenna transmitters, $K<M$ primary receivers, and random codebooks with $N=2^{B}$ precoding vectors, the mean received power at the primary receivers is approximately upper bounded by $N^{-1 / K}+\frac{\sigma^{2}}{M-1}=2^{-B / K}+\frac{\sigma^{2}}{M-1}$ with or without receiver coordination where $\sigma^{2}$ is the variance of the independent real and imaginary components of the channel estimation error. We also show that the achievable null depth is approximately lower bounded by $\frac{\sigma^{2}}{M-1}$ by considering a scenario with unquantized feedback and channel estimation error. This approximate lower bound is shown to be accurate for small $\sigma$. We present numerical results verifying the bounds and show that the channel estimation errors effectively establish a floor on the achievable performance of RVQ nullforming. The size of the RVQ codebook can be selected to approach this floor without excessive overhead.

\section{SySTEM MODEL}

We consider the MIMO system shown in Fig. 1 with an $M \geq 2$ antenna transmitter, $1 \leq K<M$ single-antenna primary receivers, and one or more secondary receivers. 


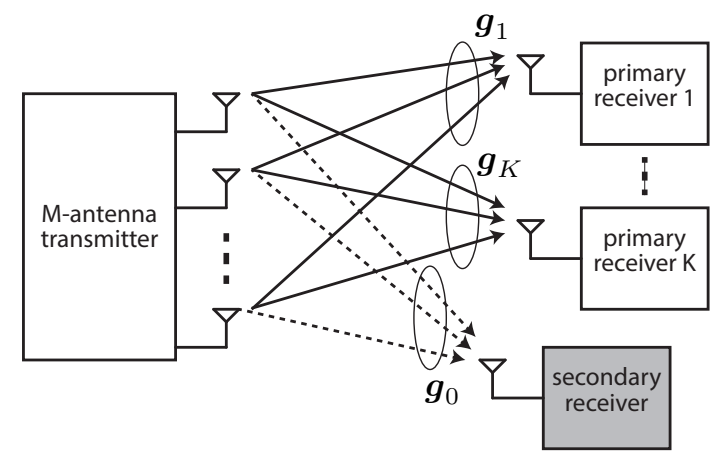

Fig. 1. MIMO system model. Only primary receivers provide feedback.

The vector channel from the transmitter to primary receiver $k$ is denoted as $\boldsymbol{g}_{k} \in \mathbb{C}^{M \times 1}$. It is assumed that $\mathrm{E}\left[\boldsymbol{g}_{k}\right]=0$ and

$$
\mathrm{E}\left[\boldsymbol{g}_{j} \boldsymbol{g}_{k}^{H}\right]= \begin{cases}2 \boldsymbol{I} & j=k \\ 0 & \text { otherwise }\end{cases}
$$

where each element of $\boldsymbol{g}_{k}$ is a proper complex Gaussian random variable with independent unit-variance real and imaginary parts. We define the normalized channels from the transmitter to primary receiver $k$ as

$$
\overline{\boldsymbol{g}}_{k}=\frac{\boldsymbol{g}_{k}}{\left\|\boldsymbol{g}_{k}\right\|} .
$$

We assume the channel estimates at the primary receivers are modeled as

$$
\widehat{\boldsymbol{g}}_{k}=\boldsymbol{g}_{k}+\boldsymbol{\epsilon}_{k}
$$

with $\epsilon_{k} \stackrel{\text { i.i.d. }}{\sim} \mathcal{C N}\left(0,2 \sigma^{2} I\right)$, with equal variance independent real and imaginary components and generated independently of the channel and precoding vector codebook. We also define the normalized estimated channels from the transmitter to primary receiver $k$ as

$$
\tilde{\boldsymbol{g}}_{k}=\frac{\widehat{\boldsymbol{g}}_{k}}{\left\|\widehat{\boldsymbol{g}}_{k}\right\|}
$$

and the normalized MIMO estimated channel matrix $\tilde{\boldsymbol{G}}=$ $\left[\tilde{\boldsymbol{g}}_{1}, \ldots, \tilde{\boldsymbol{g}}_{K}\right] \in \mathbb{C}^{M \times K}$. The primary receivers are assumed to have error-free feedback links to the transmitter for the purpose of conveying one or more precoding vector indices. These precoding vector indices are then used to compute/select a precoding vector $\boldsymbol{w} \in \mathbb{C}^{M \times 1}$ such that the interference is minimized at the primary receivers.

The channel to a secondary receiver is denoted as $\boldsymbol{g}_{0}$ and is assumed to be independent and identically distributed with respect to the primary receiver channels $\left\{\boldsymbol{g}_{k}\right\}$. Since the transmitter's precoding vector $\boldsymbol{w}$ is computed only as a function of the primary receiver channels, it is independent of $\boldsymbol{g}_{0}$. Hence $\boldsymbol{g}_{0}^{H} \boldsymbol{w}$ is equivalent in distribution to a singleantenna Rayleigh fading channel with mean received power $\mathrm{E}\left[\left|\boldsymbol{g}_{0}^{H} \boldsymbol{w}\right|^{2}\right]=2$ assuming $\boldsymbol{w}^{H} \boldsymbol{w}=1$.

\section{A. Nullforming without Primary Receiver Coordination}

If the receivers are unable to coordinate their feedback, a zero-forcing scheme similar to [8] can be used to steer nulls toward the primary receivers. Each primary receiver $k$ feeds back $B$ bits that represent a quantized version of the estimated channel's direction $\tilde{\boldsymbol{g}}_{k}$. The transmitter then computes a precoding vector that steers nulls toward all $K$ reconstructed channels.

Note that $\tilde{G}$ has at most rank $K$. This means that we can always find an $M \times(K+1)$ orthonormal matrix $\boldsymbol{A}_{\text {opt }}$ such that each $\widehat{\boldsymbol{g}}_{k}$ lies in the column space of $\boldsymbol{A}_{\text {opt }}$. If the transmitter and primary receivers somehow had access to $\boldsymbol{A}_{\text {opt }}$, each primary receiver $k$ could project $\widehat{\boldsymbol{g}}_{k}$ onto $\boldsymbol{A}_{\text {opt }}$ and quantize the unit vector $\boldsymbol{A}_{o p t}^{H} \widehat{\boldsymbol{g}}_{k} /\left\|\boldsymbol{A}_{\text {opt }}^{H} \widehat{\boldsymbol{g}}_{k}\right\|$. The transmitter would then be left with the problem of finding the null space of the $(K+1) \times K$ matrix $\boldsymbol{A}_{\text {opt }}^{H} \tilde{\boldsymbol{G}}$. Because the unit sphere in $\mathbb{C}^{K+1}$ when taking into account phase invariance (i.e., the Grassmann manifold of one dimensional subspaces) is of dimension $K$, rather than $M-1$, each primary receiver's quantizer uses $B / K$ bits per dimension instead of $B /(M-1)$ bits per dimension. This improved bit allocation reduces the channel distortion at the transmit side after reception of the feedback signals.

Although $\boldsymbol{A}_{\text {opt }}$ is not known to the transmitter or the receivers performing quantization, we can still achieve a $B / K$ bit allocation per dimension by projecting the quantization problem to an arbitrary reduced subspace. We assume each primary receiver has an independently generated codebook of unit-norm randomly generated complex vectors $\mathcal{V}_{k}=\left\{\boldsymbol{v}_{k, 1}, \ldots, \boldsymbol{v}_{k, N}\right\}$ with $\boldsymbol{v}_{k, i}=\boldsymbol{u}_{k, i} /\left\|\boldsymbol{u}_{k, i}\right\|$ and with $\left\{\boldsymbol{u}_{k, 1}, \ldots, \boldsymbol{u}_{k, N}\right\}$ all $(K+1)$-dimensional vectors consisting of independent and identically distributed zero-mean, unitvariance complex Gaussian random variables. The transmitter is assumed to know all of the codebooks.

After estimating its vector channel from the transmitter, each primary receiver first computes $\widehat{\boldsymbol{h}}_{k}=\boldsymbol{A}^{H} \widehat{\boldsymbol{g}}_{k}$ where $\boldsymbol{A} \in \mathbb{C}^{M \times(K+1)}$ is an arbitrary but common unitary matrix satisfying $\boldsymbol{A}^{H} \boldsymbol{A}=\boldsymbol{I}$ that projects the vector channels into a $K+1$ dimensional subspace. Each primary receiver then finds the index of the codebook vector that maximizes the inner product with the reduced-subspace channel, i.e.,

$$
i_{\mathrm{opt}}^{(k)}=\underset{i \in\{1, \ldots, N\}}{\operatorname{argmax}}\left|\boldsymbol{v}_{k, i}^{H} \tilde{\boldsymbol{h}}_{k}\right|^{2}
$$

where

$$
\tilde{\boldsymbol{h}}_{k}=\frac{\widehat{\boldsymbol{h}}_{k}}{\left\|\widehat{\boldsymbol{h}}_{k}\right\|}=\frac{\boldsymbol{A}^{H} \widehat{\boldsymbol{g}}_{k}}{\left\|\widehat{\boldsymbol{h}}_{k}\right\|}=\frac{\left\|\widehat{\boldsymbol{g}}_{k}\right\|}{\left\|\widehat{\boldsymbol{h}}_{k}\right\|} \boldsymbol{A}^{H} \tilde{\boldsymbol{g}}_{k} .
$$

Since $\tilde{\boldsymbol{g}}_{k}$ is isotropic and $\boldsymbol{A}$ is unitary, $\tilde{\boldsymbol{h}}_{k}$ is also isotropic. Each primary receiver then feeds back the index $i_{\mathrm{opt}}^{(k)}$ to the transmitter to facilitate computation of a zero-forcing precoding vector. Note that there are $K B$ total bits of feedback.

After receiving feedback from all of the primary receivers, the transmitter forms the matrix

$$
\boldsymbol{V}=\left[\begin{array}{lll}
\boldsymbol{v}_{1, i_{\mathrm{opt}}^{(1)}} & \cdots & \boldsymbol{v}_{K, i_{\mathrm{opt}}^{(K)}}
\end{array}\right] \in \mathbb{C}^{(K+1) \times K}
$$


from the known RVQ codebooks. Note that the rank of $\boldsymbol{V}$ is at most $K$, hence $\boldsymbol{V}^{H}$ has a nonempty nullspace. The transmitter then computes a unit-norm $M \times 1$ complex precoding vector as $\boldsymbol{w}_{\text {opt }}=\boldsymbol{A} \boldsymbol{v}_{\text {opt }}$ where $\boldsymbol{v}_{\text {opt }} \in \mathbb{C}^{K+1}$ is a unit-norm vector in the nullspace of $\boldsymbol{V}^{H}$. The vector $\boldsymbol{v}_{\text {opt }}$ can be computed, for example, by performing a singular value decomposition on $\boldsymbol{V}^{H}$. The right-singular vectors of $\boldsymbol{V}^{H}$ corresponding to the one or more singular values of $\boldsymbol{V}^{H}$ equal to zero provide an orthonormal basis for the nullspace of $\boldsymbol{V}^{H}$. Also note that $\left\|\boldsymbol{w}_{\text {opt }}\right\|=1$ since $\boldsymbol{A}^{H} \boldsymbol{A}=\boldsymbol{I}$.

\section{B. Nullforming with Primary Receiver Coordination}

In the case when the primary receivers can coordinate by exchanging channel estimates prior to sending feedback to the transmitter, we assume the transmitter and primary receivers all share a single common codebook of unitnorm randomly generated complex precoding vectors $\mathcal{W}=$ $\left\{\boldsymbol{w}_{1}, \ldots, \boldsymbol{w}_{N}\right\}$ with $\boldsymbol{w}_{i}=\boldsymbol{u}_{i} /\left\|\boldsymbol{u}_{i}\right\|$ and with $\left\{\boldsymbol{u}_{1}, \ldots, \boldsymbol{u}_{N}\right\}$ all $M$-dimensional vectors consisting of independent and identically distributed zero-mean, unit-variance complex Gaussian random variables. Note that the statistics of the codebook are the same as in the case without receiver coordination, but here each codeword is an element of $\mathbb{C}^{M}$ and all of the primary receivers share a common codebook.

After estimating the vector channels from the transmitter, the primary receivers coordinate by exchanging unquantized normalized channel estimates. One or more primary receivers then search through the common codebook to determine the index of the precoding vector that minimizes the average power over all of the primary receivers, i.e.,

$$
i_{\mathrm{opt}}=\underset{i \in\{1, \ldots, N\}}{\operatorname{argmin}} \frac{1}{K} \sum_{k=1}^{K}\left|\boldsymbol{w}_{i}^{H} \tilde{\boldsymbol{g}}_{k}\right|^{2} .
$$

Once the optimal precoding vector is found, only one primary receiver then needs to feed back the integer value of $i_{\text {opt }}$ to facilitate nullforming by the transmitter. In this case, only $B=$ $\log _{2}(N)$ bits of feedback are required. The selected precoding vector is then used directly by the transmitter.

\section{Analytical Results}

This section presents analytical results on the performance of RVQ nullforming in terms of the mean normalized received power observed by the primary receivers with and without receiver coordination as a function of the parameters $K, M$, $N$, and $\sigma^{2}$. In all cases, the precoding vector is computed from the noisy normalized channel estimates $\left\{\tilde{\boldsymbol{g}}_{k}\right\}$ whereas the performance (average normalized received power at the primary receivers) is evaluated using the normalized actual channels $\left\{\overline{\boldsymbol{g}}_{k}\right\}$.

\section{A. Approximate Lower Bound on Achievable Null Depth}

In this section, we establish an approximate lower bound on the achievable null depth of RVQ nullforming with channel estimation error by considering a system with unquantized feedback. In this case, a zero-forcing precoding vector can be calculated as

$$
\boldsymbol{w}_{\mathrm{zf}}=\alpha\left(\boldsymbol{I}-\tilde{\boldsymbol{G}}\left(\tilde{\boldsymbol{G}}^{H} \tilde{\boldsymbol{G}}\right)^{-1} \tilde{\boldsymbol{G}}^{H}\right) \boldsymbol{z}
$$

where $z$ is a randomly chosen unit-norm vector independent of $\tilde{\boldsymbol{G}}$, and $\alpha$ is a scale factor selected such that $\left\|\boldsymbol{w}_{\mathrm{zf}}\right\|^{2}=1$. We denote the normalized received power at primary receiver $k$ when the transmitter uses the precoding vector $\boldsymbol{w}_{\mathrm{zf}}$ as

$$
\nu_{\mathrm{zf}}^{(k)}=\left|\boldsymbol{w}_{\mathrm{zf}}^{H} \overline{\boldsymbol{g}}_{k}\right|^{2}=\frac{\left|\boldsymbol{w}_{\mathrm{zf}}^{H} \boldsymbol{g}_{k}\right|^{2}}{\left\|\boldsymbol{g}_{k}\right\|^{2}}
$$

and the spatially averaged normalized received power as

$$
\nu_{\mathrm{zf}}=\frac{1}{K} \sum_{k=1}^{K} \nu_{\mathrm{zf}}^{(k)} .
$$

Observe that

$$
\mathrm{E}\left[\left|\boldsymbol{w}_{\mathrm{zf}} \boldsymbol{g}_{k}\right|^{2}\right]=\mathrm{E}\left[\left|\boldsymbol{w}_{\mathrm{zf}} \boldsymbol{\epsilon}_{k}\right|^{2}\right]=2 \sigma^{2}
$$

due to the orthogonality of $\boldsymbol{w}_{\mathrm{zf}}$ and $\widehat{\boldsymbol{g}}_{k}$ and the fact that $\left\|\boldsymbol{w}_{\mathrm{zf}}\right\|^{2}=1$. Hence, the mean normalized received power of a zero-forcing nullformer with unquantized feedback can be approximated as

$\mathrm{E}\left[\nu_{\mathrm{zf}}\right]=\frac{1}{K} \sum_{k=1}^{K} \mathrm{E}\left[\frac{\left|\boldsymbol{w}_{\mathrm{zf}}^{H} \boldsymbol{\epsilon}_{k}\right|^{2}}{\left\|\boldsymbol{g}_{k}\right\|^{2}}\right] \approx 2 \sigma^{2} \mathrm{E}\left[\frac{1}{\left\|\boldsymbol{g}_{k}\right\|^{2}}\right]=\frac{\sigma^{2}}{M-1}$

where the approximation results from an assumption that $\left|\boldsymbol{w}_{\mathrm{zf}}^{H} \boldsymbol{\epsilon}_{k}\right|^{2}$ and $\left\|\boldsymbol{g}_{k}\right\|^{2}$ are independent and the final equality results from the fact that $1 /\left\|\boldsymbol{g}_{k}\right\|^{2}$ is inverse Chi-squared distributed with $2 M$ degrees of freedom. Numerical results in Section IV show that this approximation is accurate for small values of $\sigma^{2}$ but is somewhat pessimistic for larger values of $\sigma^{2}$. This can be understood intuitively by considering the case when $\boldsymbol{g}_{k}$ is very small with respect to $\boldsymbol{\epsilon}_{k}$. In this case, $\widehat{\boldsymbol{g}}_{k} \approx \boldsymbol{\epsilon}_{k}$ and consequently $\boldsymbol{w}_{\mathrm{zf}}^{H} \boldsymbol{\epsilon}_{k} \approx 0$.

\section{B. Nullforming without Primary Receiver Coordination}

In the absence of receiver coordination, each primary receiver finds the codebook vector that maximizes the inner product with the receiver's reduced-subspace estimated channel. The normalized received power at primary receiver $k$ when the transmitter uses the precoding vector $\boldsymbol{w}_{\mathrm{opt}}$ is

$$
\nu_{\mathrm{opt}}^{(k)}=\left|\boldsymbol{w}_{\mathrm{opt}}^{H} \overline{\boldsymbol{g}}_{k}\right|^{2}
$$

and the spatially averaged normalized received power is

$$
\nu_{\mathrm{opt}}=\frac{1}{K} \sum_{k=1}^{K} \nu_{\mathrm{opt}}^{(k)} .
$$

For small $\sigma^{2}$, we can write

$$
\left|\boldsymbol{w}_{\mathrm{opt}}^{H} \overline{\boldsymbol{g}}_{k}\right|^{2}=\frac{\left.\mid \boldsymbol{w}_{\mathrm{opt}}^{H}\left(\widehat{\boldsymbol{g}}_{k}-\boldsymbol{\epsilon}_{k}\right)\right)\left.\right|^{2}}{\left\|\boldsymbol{g}_{k}\right\|^{2}} \approx\left|\boldsymbol{w}_{\mathrm{opt}}^{H} \tilde{\boldsymbol{g}}_{k}\right|^{2}+\frac{2 \sigma^{2}}{M-1}
$$

where the approximation results from the assumption that $\left|\boldsymbol{w}_{\mathrm{opt}}^{H} \boldsymbol{\epsilon}_{k}\right|^{2}$ and $\left\|\boldsymbol{g}_{k}\right\|^{2}$ are independent. An upper bound on 
$\nu_{\text {opt }}$ for the case without channel estimation error was developed in [19, Thm. 1]. This result can be used to establish an approximate upper bound on the mean normalized received power without receiver coordination for the case with channel estimation error as

$$
\begin{aligned}
\mathrm{E}\left[\nu_{\mathrm{opt}}\right] & \lesssim \frac{K+1}{M} N \beta\left(N, \frac{K+1}{K}\right)+\frac{2 \sigma^{2}}{M-1} \\
& \leq N^{-1 / K}+\frac{2 \sigma^{2}}{M-1}
\end{aligned}
$$

where $\beta(s, t)$ denotes the beta function defined as

$$
\beta(s, t)=\frac{\Gamma(s) \Gamma(t)}{\Gamma(s+t)} \text { with } \Gamma(x)=\int_{0}^{\infty} t^{x-1} e^{-t} d t .
$$

\section{Nullforming with Primary Receiver Coordination}

In the case with receiver coordination, the primary receivers exchange channel estimates and find the codebook vector that minimizes the spatially averaged normalized received power. The average normalized actual received power when the transmitter uses precoding vector $\boldsymbol{w}_{i}$ can be written as

$$
\nu_{i}=\frac{1}{K} \sum_{k=1}^{K}\left|\boldsymbol{w}_{i}^{H} \overline{\boldsymbol{g}}_{k}\right|^{2}
$$

The minimum average normalized received power over the common $N$-vector RVQ codebook is then

$$
\nu_{\min }=\min _{i \in\{1, \ldots, N\}} \nu_{i}
$$

Similar to the case without receiver coordination, we have

$$
\left|\boldsymbol{w}_{i}^{H} \overline{\boldsymbol{g}}_{k}\right|^{2}=\frac{\left.\mid \boldsymbol{w}_{i}^{H}\left(\widehat{\boldsymbol{g}}_{k}-\boldsymbol{\epsilon}_{k}\right)\right)\left.\right|^{2}}{\left\|\boldsymbol{g}_{k}\right\|^{2}} \approx\left|\boldsymbol{w}_{i}^{H} \tilde{\boldsymbol{g}}_{k}\right|^{2}+\frac{2 \sigma^{2}}{M-1}
$$

for small $\sigma^{2}$. An upper bound on $\nu_{\mathrm{opt}}$ for the case without channel estimation error was developed in [19, Thm. 2]. This result can be used to establish an approximate upper bound on the mean normalized received power without receiver coordination for the case with channel estimation error as

$$
\begin{aligned}
\mathrm{E}\left[\nu_{\min }\right] & \lesssim N \beta\left(N, \frac{K+1}{K}\right)+\frac{2 \sigma^{2}}{M-1} \\
& \leq N^{-1 / K}+\frac{2 \sigma^{2}}{M-1}
\end{aligned}
$$

with $\beta(s, t)$ as defined in (5).

\section{Remark}

For large codebooks with $N \rightarrow \infty$, the results in the previous sections imply

$$
\frac{\sigma^{2}}{M-1} \lesssim \mathrm{E}[\nu] \lesssim \frac{2 \sigma^{2}}{M-1}
$$

where $\nu=\nu_{\min }$ or $\nu=\nu_{\mathrm{opt}}$, the approximate lower bound is from the unquantized feedback analysis, and the approximate upper bound is from (3) and (8).

\section{NuMERICAL RESUltS}

In this section, Monte-Carlo simulation results with 1000 iterations are plotted against the analytical results from Section III. In each iteration of the Monte-Carlo simulation, the channel vectors $\boldsymbol{g}_{k} \in \mathbb{C}^{M}$ are drawn i.i.d. from $\mathcal{C N}(0,2 \boldsymbol{I})$, with unit variance independent real and imaginary components, and each normalized precoding vector codebook is also randomly generated, independently of the channel.

Fig. 2 shows the mean normalized received power, averaged over the channel and codebook realizations, for a $K=3$ primary receiver system with $M=8$ transmit antennas and $N=2^{B}$ precoding vectors per codebook. Results are plotted with and without channel estimation errors where the case with channel estimation error assumes $\sigma^{2}=0.05$. We see that the approximate upper bounds developed in Section III are both somewhat loose but provide the correct scaling for the RVQ results. This example confirms that receiver coordination tends to achieve better nullforming performance and also demonstrates that the RVQ performance (both with and without receiver coordination) can approach the approximate lower bound corresponding to unquantized feedback for moderate values of $B$.

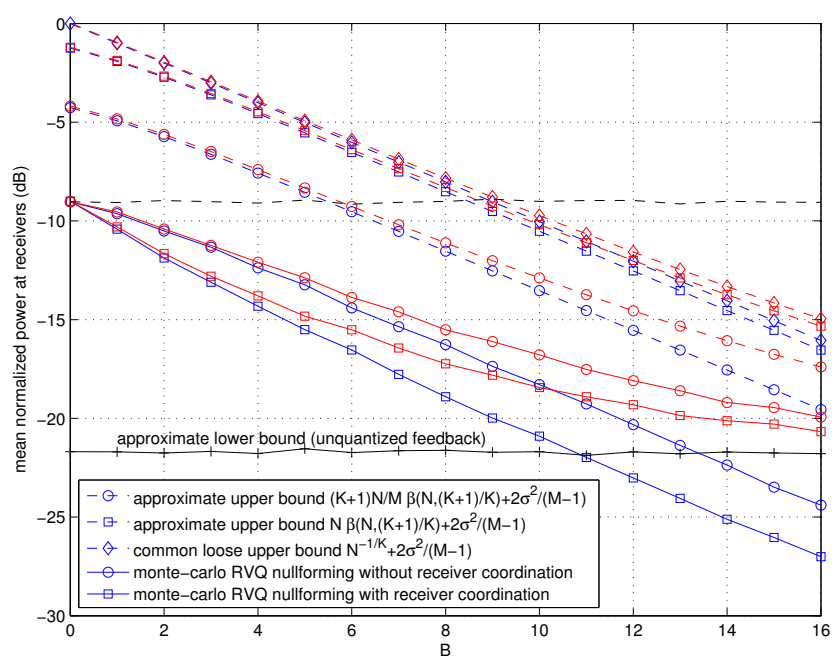

Fig. 2. Mean normalized received power $\mathrm{E}\left[\nu_{\min }\right]$ and $\mathrm{E}\left[\nu_{\mathrm{opt}}\right]$ for a $K=3$ primary receiver system with $M=8$ transmit antennas with and without receiver coordination, as a function of the codebook size $2^{B}$. Blue lines use $\sigma^{2}=0$ (no channel estimation error) and red lines use $\sigma^{2}=0.05$.

Figure 3 shows the mean normalized received power as a function of the channel estimation error standard deviation $\sigma$ and $B$ with $M=8$ and $K=3$. These results show that the approximate lower bound developed in Section III-A is accurate for small values of $\sigma$. We also see that there is a significant gap in the performance between RVQ nullforming and the unquantized feedback approximate lower bound when $\sigma$ is small. As $\sigma$ becomes large, there is little benefit in using large RVQ codebooks and/or receiver coordination.

Fig. 4 shows the mean normalized received power as a function of the number of primary receivers $K$ for different values of $B$ with $M=8$ and $\sigma^{2}=0.05$. These results 


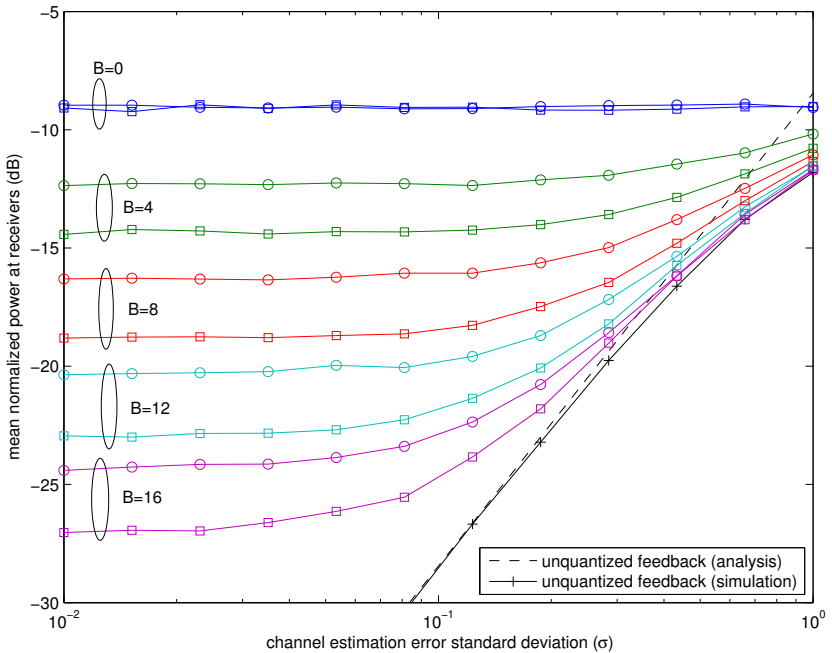

Fig. 3. Mean normalized received power $\mathrm{E}\left[\nu_{\mathrm{min}}\right]$ and $\mathrm{E}\left[\nu_{\mathrm{opt}}\right]$ for a $M=8$ transmit antenna and $K=3$ receiver system with and without receiver coordination, respectively, as a function of the channel estimation error standard deviation $\sigma$ and the codebook size $2^{B}$. Lines with squares and circles are results with and without receiver coordination, respectively.

show that RVQ nullforming can approach the approximate lower bound for moderate values of $B$ when $K$ is small, but significantly larger codebooks are required for larger values of $K$ due to the $N^{-1 / K}$ scaling. For larger values of $K$, the benefits of receiver coordination are also more evident where, for example, the $B=12$ system with receiver coordination outperforms the $B=16$ system without receiver coordination.

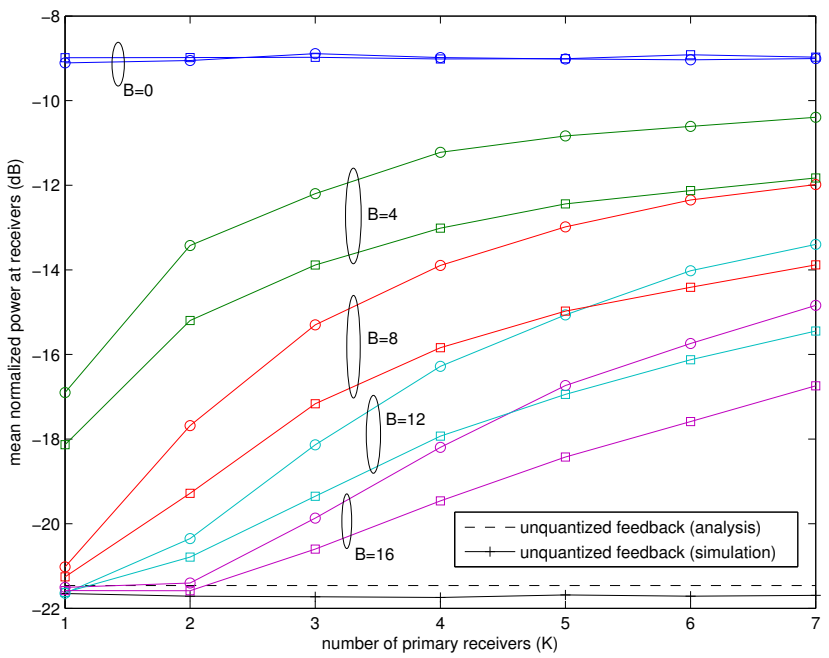

Fig. 4. Mean normalized received power $\mathrm{E}\left[\nu_{\mathrm{min}}\right]$ and $\mathrm{E}\left[\nu_{\mathrm{opt}}\right]$ for a $M=8$ transmit antenna system with and without receiver coordination, respectively, as a function of the number of primary receivers $K$ and the codebook size $2^{B}$. The channel estimation error variance is fixed at $\sigma^{2}=0.05$. Lines with squares and circles are results with and without receiver coordination, respectively.

\section{CONClusion}

This paper analyzes the performance of MIMO nullforming with RVQ limited feedback in single-stream systems with and without receiver coordination and with channel estimation error. Approximate upper and lower bounds are developed for the mean received power at the primary receivers with and without receiver coordination. Numerical results confirm the analysis and show that the channel estimation errors effectively establish a floor on the achievable performance of RVQ nullforming. The size of the RVQ codebook can be selected to approach this floor without excessive overhead.

\section{REFERENCES}

[1] D. Gerlach and A. Paulraj, "Adaptive transmitting antenna arrays with feedback," IEEE Sig. Proc. Lett., vol. 1, no. 10, pp. 150-152, 1994.

[2] D. J. Love, R. W. Heath Jr, W. Santipach, and M. L. Honig, "What is the value of limited feedback for MIMO channels?" IEEE Comm. Mag., vol. 42, no. 10, pp. 54-59, 2004.

[3] D. J. Love, R. W. Heath, Jr., V. Lau, D. Gesbert, B. D. Rao, and M. Andrews, "An overview of limited feedback in wireless communication systems," IEEE Jour. Select. Areas in Comm., vol. 26, no. 8, pp. 13411365, Oct. 2008.

[4] T. Yoo, N. Jindal, and A. Goldsmith, "Multi-antenna downlink channels with limited feedback and user selection," Selected Areas in Communications, IEEE Journal on, vol. 25, no. 7, pp. 1478-1491, 2007.

[5] D. J. Love, R. W. Heath Jr., and T. Strohmer, "Grassmannian beamforming for multiple-input multiple-output wireless systems," IEEE Trans. Info. Th., vol. 49, no. 10, pp. 2735-2747, Oct. 2003.

[6] J. C. Roh and B. D. Rao, "Efficient feedback methods for MIMO channels based on parameterization," IEEE Trans. Wireless Comm., vol. 6, no. 1, pp. 282-292, Jan. 2007.

[7] V. Raghavan, R. Heath, Jr., and A. Sayeed, "Systematic codebook designs for quantized beamforming in correlated MIMO channels," IEEE Jour. Select. Areas in Comm., vol. 25, no. 7, pp. 1298-1310, Sep. 2007.

[8] N. Jindal, "MIMO broadcast channels with finite-rate feedback," Information Theory, IEEE Trans. on, vol. 52, no. 11, pp. 5045-5060, 2006.

[9] P. Ding, D. J. Love, and M. D. Zoltowski, "Multiple antenna broadcast channels with shape feedback and limited feedback," IEEE Trans. Sig. Proc., vol. 55, no. 7, pp. 3417-3428, July 2007.

[10] C. K. Au-Yeung, D. J. Love, and S. Sanayei, "Trellis coded line packing: Large dimensional beamforming vector quantization and feedback transmission," IEEE Trans. Wireless Comm., vol. 10, no. 6, pp. 1844-1853, June 2011.

[11] W. Santipach and M. L. Honig, "Asymptotic performance of mimo wireless channels with limited feedback," in IEEE Military Communications Conf., 2003. MILCOM'03, vol. 1. IEEE, 2003, pp. 141-146.

[12] _ - "Asymptotic capacity of beamforming with limited feedback," in Information Theory, 2004. ISIT 2004. Proceedings. International Symposium on. IEEE, 2004, p. 290.

[13] W. Dai, Y. Liu, and B. Rider, "Quantization bounds on grassmann manifolds and applications to mimo communications," Information Theory, IEEE Transactions on, vol. 54, no. 3, pp. 1108-1123, 2008.

[14] W. Santipach and M. Honig, "Capacity of a multiple-antenna fading channel with a quantized precoding matrix," Information Theory, IEEE Transactions on, vol. 55, no. 3, pp. 1218-1234, 2009.

[15] D. Ryan, "Performance of RVQ limited feedback beamforming over correlated channels," in Proc. IEEE Wireless Comm. and Net. Conf., April 2010.

[16] W. Santipach and K. Mamat, "Tree-structured random vector quantization for limited-feedback wireless channels," IEEE Trans. Wireless Comm., vol. 10, no. 9, pp. 3012-3019, Sept. 2011.

[17] V. Raghavan and V. V. Veeravalli, "Ensemble properties of RVQ-based limited feedback beamforming codebooks," IEEE Trans. Info. Th., 2012, submitted available http://arxiv.org/abs/1207.1524.

[18] C. K. Au-Yeung and D. Love, "On the performance of random vector quantization limited feedback beamforming in a miso system," Wireless Communications, IEEE Trans. on, vol. 6, no. 2, pp. 458-462, 2007.

[19] D.R. Brown III and D. Love, "On the performance of MIMO nullforming with random vector quantization limited feedback," IEEE Transactions on Wireless Communications, vol. 13, no. 5, pp. 2884-2893, May 2014. 